\title{
SUMO-1 modification of centrosomal protein hNinein promotes hNinein nuclear localization
}

\author{
Tai-Shan Cheng ${ }^{a, 1}$, Li-Kwan Chang ${ }^{\text {b,1 }}$, Shen-Long Howng ${ }^{c}$, \\ Pei-Jung Lu ${ }^{\mathrm{d}}$, Chu-I Lee ${ }^{\mathrm{e}}$, Yi-Ren Hong ${ }^{\text {a,* }}$

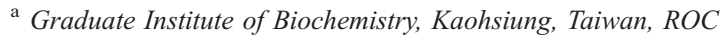 \\ ${ }^{\mathrm{b}}$ Department of Biology, Kaohsiung, Taiwan, ROC \\ ${ }^{\mathrm{c}}$ Neurosurgery Department of Kaohsiung Medical University, Kaohsiung, Taiwan, ROC \\ ${ }^{\mathrm{d}}$ Kaohsiung Veterans General Hospital Kaohsiung, Kaohsiung, Taiwan, ROC \\ e Department of Medical Technology, Fooyin University; Kaohsiung, Taiwan, ROC
}

Received 25 February 2005; accepted 18 June 2005

\begin{abstract}
A centrosomal-associated protein, ninein is a microtubules minus end capping, centrosome position, and anchoring protein, but the underlying structure and physiological functions are still unknown. To identify the molecules that regulate the function of human ninein in centrosome, we performed yeast two-hybrid screen and isolated the SUMO-conjugating E2 enzyme, Ubc9, and SUMOylation enhancing enzymes, including PIAS1 and PIASx $\alpha$, as binding partners of hNinein. These interactions as well as the interaction between hNinein and SUMO-1 are also confirmed by a glutathione $S$-transferase (GST) pull-down experiment. Furthermore, the C-terminal region of hNinein can be SUMOylated in vitro and in HeLa cells transfected with a plasmid expressing GFP-hNinein. Our findings firstly place SUMOylation target on the centrosome structure protein, hNinein, which results in the switch localization from centrosome to nucleus, suggesting the importance of the SUMOylation of hNinein and probably other centrosomal proteins may also be involved in the centrosome activity.
\end{abstract}

(C) 2005 Elsevier Inc. All rights reserved.

Keywords: hNinein; Centrosome; SUMOylation

\section{Introduction}

The centrosome plays key roles in the formation of the mitotic spindle, cell polarity, and cell locomotion. In a typical somatic cell, the centrosome is composed of a pair of centrioles that are surrounded by a mass of amorphous pericentriolar material $(\mathrm{PCM})$. The recent identification of molecular components shows that PCM may be involved in the formation of the complex of $\gamma$-tubulin (Zheng et al.,

\footnotetext{
Abbreviations: Ubc9, ubiquitin-conjugation enzyme 9; PIAS1, protein inhibitor of activated STAT 1; SUMO-1, small ubiquitin-related modifier-1; RanGAP1, Ran-GTPase-activating protein 1; GSK3 $\beta$, Glycogen synthase kinase 3 beta; RING domain, RING finger domain; aa., amino acid.

* Corresponding author. Graduate Institute of Biochemistry, Kaohsiung Medical University, No. 100, Shih-Chuan 1st Road, Kaohsiung 80708 Taiwan, ROC. Tel.: +886 7 3121101x5386; fax: +886 73218309 .

E-mail address: m835016@cc.kmu.edu.tw (Y.-R. Hong).

${ }^{1}$ Both authors contributed equally to the work.
}

1991; Fuller et al., 1995), centrin (Moudjou et al., 1991; Baron et al., 1992; Middendorp et al., 1997), pericentrin (Doxsey et al., 1994) and ninein (Bouckson-Castaing et al., 1996; Dictenberg et al., 1998; Mack et al., 1998; Mogensen, 1999; Hong et al., 2000a; Piel et al., 2000; Bornens, 2002, which are organized into a highly ordered lattice (Piel et al., 2000; Doxsey, 2001).

The first identified target for SUMO-1 modification was RanGAP1 (Matunis et al., 1996; Mahajan et al., 1997). Posttranscriptional modification by SUMO is known to influence protein interactions, nuclear localization, and stability (Ahn et al., 2001; Joseph et al., 2002; Pichler et al., 2002; Su and Li, 2002; Marx, 2005). This modification process involves the function of a SUMO-activating E1 enzyme and the SUMO-conjugating E2 enzyme. The E1 enzyme, a heterodimeric protein consisting of a 38-kDa Aos1 and a $72-\mathrm{kDa}$ Uba2 subunits, cleaves the four C-terminal amino acid residues from SUMO, which is a prerequisite for 
SUMO conjugation (Gong et al., 1999). The SUMOconjugating enzyme E2, also referred to as Ubc9, is required for linking SUMO to its target proteins (Schwarz et al., 1998). PIAS (protein inhibitor of activated STAT) family, on the other hand, although is not required for SUMOylation, enhances protein SUMOylation (Johnson and Gupta, 2001; Kahyo et al., 2001; Marx, 2005).

Human ninein (hNinein) was originally isolated by the yeast two-hybrid system with the GSK3 3 as a bait (Hong et al., 2000a). Molecular characterization shows that hNinein is oligomerized at the C-terminal end which overlapped with GSK3 $\beta$ binding site, suggesting that oligomerization of hNinein may be regulated by GSK $3 \beta$ phosphorylation (Hong et al., 2000a,b). In a search for proteins that regulate the functions of hNinein in centrosome, we identified Ubc9, PIAS1, and PIASx $\alpha$ as binding partners of hNinein. In the present study, we show that hNinein is capable of binding to SUMO-1 and is SUMOylated in vitro and in vivo. In addition, overexpression SUMO-1 also causes endogenous hNinein; $\gamma$ tubulin and pericentrin were translocated into nucleus. These results indicate SUMOylation of centrosome structure protein that promotes nucleocytoplasmic exchange, suggesting that SUMO-1 could provide a common feature to direct centrosomal structure proteins to switch localization, may be involved in the centrosome activity.

\section{Materials and methods}

\section{Plasmid construction}

Plasmids pcDNA-Ubc9, pcDNA-SUMO-1, pGEX-4T1, and pGEX-Ubc9, which express HA-tagged Ubc9, SUMO-1, GST, and GST-Ubc9, respectively, were provided by Van G. Wilson (Rangasamy et al., 2000). Plasmid pET-SUMO-1 which was used to express His-tagged SUMO-1 in E. coli BL21(DE3) and pDsRed-SUMO-1 which was to express RFP-SUMO-1 in cells were previously constructed (Chang et al., 2004). Plasmid pCR-SUMO-1, which encodes FLAGtagged SUMO-1, was constructed by inserting a PCRamplified SUMO-1 DNA fragment into pCR3.1 (Invitrogen) (Chang et al., 2004). Plasmid pGEX-SUMO-1, obtained from Frauke Melchior (Mahajan et al., 1998), encodes a GSTSUMO-1 fusion protein lacking the four C-terminal amino acids of SUMO-1. The SUMO-1 gene fragment in pGEXSUMO-1 was isolated by Bam HI digestion and inserted into the Bam HI site of pACT2 to generate pACT2-SUMO-1. To construct plasmids, pET32a-C-terminal hNinein (aa. 16172090) and pET32a-N-terminal hNinein (aa. 1-471), for expressing His-tagged hNinein in E coli BL21(DE3), DNA fragments encoding the $\mathrm{C}$-terminal region of $\mathrm{hNinein}$ were amplified by PCR, using primers containing sense sequences, 5'-CGGGATCCATGCTATGCCAGAAGGAA, and antisense sequences, 5'-CCGCTCGAGTTAATGGCAATAAAGGGATG. A DNA fragment encoding the N-terminal hNinein was amplified by PCR, using primers containing sense sequences: 5'-CGGGATCCATGGATGAGGTGGAGCAG and antisense sequences, 5'-CCGCTCGAGAAGGCGGT-
CCCGGATATA. Boldfaced letters indicate BamHI and XhoI restriction enzyme sites. The PCR fragments were then inserted into the Bam HI-XhoI sites in pET-32a(+) (Novagen). A DNA fragment encoding C-terminal hNinein (aa. 1617-2090 and 1719-2090) was inserted into the Bam HI-XhoI sites of pAS21 and pcDNA 3.1 vector. The full-length hNinein gene was constructed to pEGFP C2 vector (Clontech) and it was fused at the restriction sites $B g l \mathrm{II}$ and $\mathrm{SalI}$.

\section{Yeast two-hybrid system}

Standard techniques were used for the yeast two-hybrid system (Fields and Song, 1989; Chien et al., 1991; Zhu, 1997). Briefly, hNinein gene (aa. 1617-1931) was cloned in frame with the GAL4 DNA binding domain (GAL4 BD) in the pAS2-1 vector (MARCHMAKER Two-Hybrid System; Clontech, USA) to yield pAS2-1-C-hNinein. A human testis cDNA library (Clontech) was screened by co-transforming yeast YRG-2 (Stratagene) with pAS2-1-C-hNinein bait plasmid DNA (GAL4 BD) and human testis library plasmid DNA (GAL4 AD). Positive clones were selected based on the ability of the cells to grow on Trp, Leu, and His dropout media supplemented with 3-aminotriazole (3-AT, and an inhibitor of HIS3), and blue colony color in $\beta$-galactosidase filter assay. Yeast strain Y190 was co-transformed with GAL4 BD and GAL4 AD to confirm to positive phenotypes. $\beta$-Galactosidase activity was determined in Z-buffer (150 $\mathrm{mM}$ phosphate buffer, $\mathrm{pH} 7.0,10 \mathrm{mM} \mathrm{KCl}$, and $1 \mathrm{mM}$ $\mathrm{MgSO}_{4}$ ) containing $4 \mathrm{mg} / \mathrm{ml}$ of O-nitrophenyl- $\beta$-D-galactopyranoside. Reactions were carried out at $30{ }^{\circ} \mathrm{C}$, stopped by the addition of $250 \mathrm{mM}$ sodium carbonate, and enzyme activity was determined by measuring absorbance at a wavelength of $420 \mathrm{~nm}$.

\section{GST-pull-down assay}

E. coli BL21(DE3)(pGEX-Ubc9) or BL21(DE3)(pGEXPIAS1) or BL21(DE3)(pGEX-SUMO-1) was cultured in 3 $\mathrm{ml}$ of $\mathrm{LB}$ medium at $37{ }^{\circ} \mathrm{C}$ to the mid-log phase. Isopropylthio- $\beta$-D-galactoside (IPTG) was then added to a final concentration of $1 \mathrm{mM}$ to induce the expression of GST fusion proteins. After culturing for $3 \mathrm{~h}$, cells were pelleted by centrifugation and suspended in $100 \mu \mathrm{l}$ of a lysis buffer, B-Per (Pierce, Rockford, IL), containing 10 $\mu l$ leupeptin, aprotinin, and 4-(2-aminoethyl)-benzenesulfonyl fluoride. The suspension was centrifuged again at 10,000 rpm for $5 \mathrm{~min}$ at $4{ }^{\circ} \mathrm{C}$ with a T15A22 rotor in a HITACHI CF R15 centrifuge. Glutathione-Sepharose 4B beads $(20 \mu \mathrm{l})$ (Amersham Pharmacia Biotech) were then added to the supernatant, and the mixture was incubated under shaking for $1 \mathrm{~h}$ at $4{ }^{\circ} \mathrm{C}$. The beads were washed three times with NETN buffer (20 mM Tris-HCl, pH 8.0, $100 \mathrm{mM} \mathrm{NaCl}, 1$ $\mathrm{mM}$ EDTA, 0.5\% NP-40). After washing, the beads were added to the lysate $(300 \mu \mathrm{l})$ prepared from E. coli lysate containing His-tagged $\mathrm{C}$-terminal hNinein. The reaction mixture was incubated on ice for $1 \mathrm{~h}$ to allow the binding between a GST-fusion protein, including GST-Ubc9, or 
GST-PIAS1, or GST-SUMO-1, and His-tagged C-terminal hNinein. The beads were subsequently washed with NETN buffer. An equal volume of $2 \times$ electrophoresis sample buffer was then added to the mixture and proteins were extracted from the beads by heating at $95{ }^{\circ} \mathrm{C}$ for $5 \mathrm{~min}$. Proteins were finally analyzed by SDS-polyacrylamide gel electrophoresis and immunoblotting.

\section{Immunoblot analysis}

Proteins resolved by SDS-polyacrylamide gel were electrotransferred to Hybond C membrane (Amersham) at $90 \mathrm{~V}$ for 1 $\mathrm{h}$ and probed with the appropriate antibodies. The SuperSignal West Pico chemiluminescent substrate (Pierce) was used to visualize the proteins on the membrane.

\section{Immunoprecipitation}

HEK293 cells $\left(1 \times 10^{7}\right)$ transfected with pcDNA-Cterminal hNinein and pCR-SUMO-1 were washed with phosphate-buffered saline (PBS). The lysate was prepared by adding $1 \mathrm{ml}$ of radioimmune precipitation assay buffer (50 mM Tris-HCl, pH 7.8, $150 \mathrm{mM} \mathrm{NaCl}, 5 \mathrm{mM}$ EDTA, $0.5 \%$ Triton X-100, $0.5 \%$ Nonidet P-40, $0.1 \%$ deoxycholate, and $10 \mu \mathrm{g} / \mathrm{ml}$ each of leupeptin, aprotinin, and 4-(2aminoethyl)benzenesulfonyl fluoride) to the cells. Then the lysate was centrifuged with a microcentrifuge at $10,000 \times g$ for $20 \mathrm{~min}$. The supernatant was added with anti-HA (1:500 dilution) (Roche Applied Science) antibody at $4{ }^{\circ} \mathrm{C}$ for $1 \mathrm{~h}$. Protein-A/G-agarose beads $(30 \mu \mathrm{l})$ (Oncogene, Boston, MA) were added to the lysate, and the mixture was incubated under shaking for $1 \mathrm{~h}$ at $4{ }^{\circ} \mathrm{C}$. The beads were finally collected by centrifugation and washed three times with radioimmune precipitation assay buffer. Proteins binding to the beads were eluted by adding $20 \mu \mathrm{l}$ of $2 \times$ electrophoresis sample buffer and analyzed by immunoblotting with anti-HA antibody. After immunoprecipitation, proteins were pooled and separated by SDS-polyacrylamide gel electrophoresis.

\section{In vitro SUMO conjugation}

Bacterially expressed His-tagged C-terminal hNinein, Histagged N-terminal hNinein and His-tagged SUMO-1 lacking the C-terminal glycine residues were purified according the method described for the purification of GST-Ubc9. SUMO E1 was purchased from LAE biotechnology Co. (Taipei, Taiwan). Each SUMO-conjugation reaction contained $3 \mu \mathrm{g}$ of His-tagged hNinein, $500 \mathrm{ng}$ each of GST-Ubc9 and SUMO$1,15 \mathrm{ng}$ of SUMO E1, and $4 \mu \mathrm{l}$ of ATP-regenerating buffer (200 mM Tris-HCl, pH 7.8, $20 \mathrm{mM} \mathrm{MgCl}, 20 \mathrm{mM}$ ATP, 4 $\mathrm{mM}$ DTT, and $800 \mu \mathrm{M}$ NEM). The final reaction volume was $16 \mu \mathrm{l}$. The reaction mixtures were incubated at $37{ }^{\circ} \mathrm{C}$ for $2 \mathrm{~h}$. After incubation, $16 \mu \mathrm{l}$ of $2 \times$ electrophoresis sample buffer was added to the reaction mixtures, which were then heated at $95{ }^{\circ} \mathrm{C}$ for $5 \mathrm{~min}$. Proteins in the mixtures were resolved by SDS-polyacrylamide gel electrophoresis. His- tagged hNinein was then detected by immunoblotting with anti-His antibody.

\section{Site-directed mutagenesis}

Site-directed mutagenesis experiments to make hNinein mutants were carried out according to the manufacturer's directions (Stratagene). HA-tagged C-terminal hNinein K1641R mutant was amplified by PCR, using sense (5'CAAGAGAAGTTTAATCTGAGAGAAGAACTGGAACGTTGTAAAG) and antisense (5'CTTTACAACGTTCCAGTTCTTCTCTCAGATTAAACTTCTCTTC) as primers and pcDNA-C-terminal hNinein as template. HA-tagged Cterminal hNinein K1641R/K1680R double mutant was amplified by PCR, using sense (5'CAGGAAAACCACCTTCTCAGAGATGAACTGGAGAAAATG) and antisense (5'CATTTTCTCCAGTTCATCTCTGAGAAGGTGGTTTTCCTG) as primers and pcDNA-C-terminal hNinein K1641R mutant as template. All mutants were sequenced to confirm that only the intended point mutations were introduced.

\section{Cell culture, transfections and fluorescence microscopy}

HeLa cells were grown at $37{ }^{\circ} \mathrm{C}$ in DMEM supplemented with $10 \%$ FBS and penicillin-streptomycin $(100 \mathrm{IU} / \mathrm{ml})$. For transient transfection studies, HeLa cells were seeded onto glass coverslips at a density of $0.7 \times 10^{5}$ cells per well in a 24 well plate. Cells in each well were transfected with $1 \mu \mathrm{g}$ pEGFP-hNinein and/or pDsRed-SUMO-1 DNA, using Lipofectamine plus reagent (Life Technologies). Cells were washed with PBS and fixed with 3\% FA in PBS. The fixed cell were probed with anti- $\boldsymbol{\gamma}$-tubulin monoclonal antibody (Sigma), antipericentrin polyclonal antibody (Covance) or affinity-purified anti-hNinein antibody (Chen et al., 2003). Finally, cells mounted in DAKO fluorescence mounting medium (DAKO), and DNA was stained with DAPI (Roche). Cells were imaged on OLYMPUS confocal laser scanning microscope.

\section{Results}

hNinein interacts with Ubc9 and PIAS in yeast

Human ninein was originally isolated by a yeast two-hybrid screen, using GSK $3 \beta$ as a bait (Hong et al., 2000a). To identify proteins that interact with hNinein, a yeast two hybrid screen was performed, using pAS2-1-C-hNinein (encoding amino acids 1617-2090 of hNinein; GenBank Accession No. AF302773) as bait and a testis cDNA library constructed in pACT2 as prey (Clontech). Among $2 \times 10^{6}$ transformants screened, 54 clones were identified that were positive for the expression of the selection markers (LacZ and HIS3). The pACT2 plasmid containing the cDNA sequence of hNineininteracting partners was recovered from the yeast cells. Blast searches against GenBank database revealed that these clones contained sequences of Ubc9 (158 amino acids, GenBank Accession No. NM_003345) and two putative E3 ligases, PIAS1 (651 amino acids, GenBank Accession No. AF167160), 
and PIASx $\alpha$ (572 amino acids, GenBank Accession No. AF077953) (Fig. 1A). A clone containing the RING domain of PIASx $\alpha$ was also obtained (Fig. 1A), indicating that the RING domain of PIAS is necessary for the interaction with hNinein.

\section{hNinein interacts with SUMO-1 in yeast}

Ubc9 is a SUMO-conjugating E2 enzyme transferring SUMO-1 to a target protein. The mammalian PIAS family of proteins, including PIAS1, PIASx $\alpha$, PIASx $\beta$ and PIASy, has been reported to have SUMO-1 ligase activities toward various target proteins (Schwarz et al., 1998; Johnson and Gupta, 2001). To explore whether hNinein might also be a target protein for SUMOylation, we first examined whether interaction between hNinein and SUMO-1 could be detected in the yeast system. To this end, pACT2-Ubc9, pACT2-PIAS1, pACT2-PIASx $\alpha$ and pACT2-SUMO-1 (Fig. 1B) were cotransformed with pAS2-1$\mathrm{C}$-hNinein into yeast strain Y190 and assayed for $\beta$-galactosidase activity and histidine autotrophy. Indeed, the hNinein also interacted with SUMO-1 (Fig. 1B).
hNinein interacts with Ubc9, PIAS1 and SUMO-1 in vitro

To further confirm that hNinein interacts with Ubc9, PIAS1 and SUMO-1, GST-fusion proteins, including GST-Ubc9, GST-PIAS1 and GST-SUMO-1, were expressed, purified, and incubated with His-tagged C-terminal hNinein (aa. 16172090). After extensive washing, the proteins bound to glutathione-sepharose beads were separated by SDS-PAGE and detected by Western blotting. The result revealed that Histagged C-terminal hNinein was retained by GST-Ubc9glutathione-sepharose beads (Fig. 1C, lane 3), GSTPIAS1-glutathione-sepharose beads (Fig. 1C, lane 4) and GST-SUMO-1-glutathione-sepharose beads (Fig. 1C, lane 5), but not GST alone (Fig. 1C, lane 2), indicating that hNinein indeed interacts with Ubc9, PIAS1 and SUMO-1.

\section{C-terminal of hNinein is conjugated with SUMO-1 in vitro and in vivo}

An in vitro assay was performed to examine whether hNinein is covalently modified by SUMO-1. In this assay,
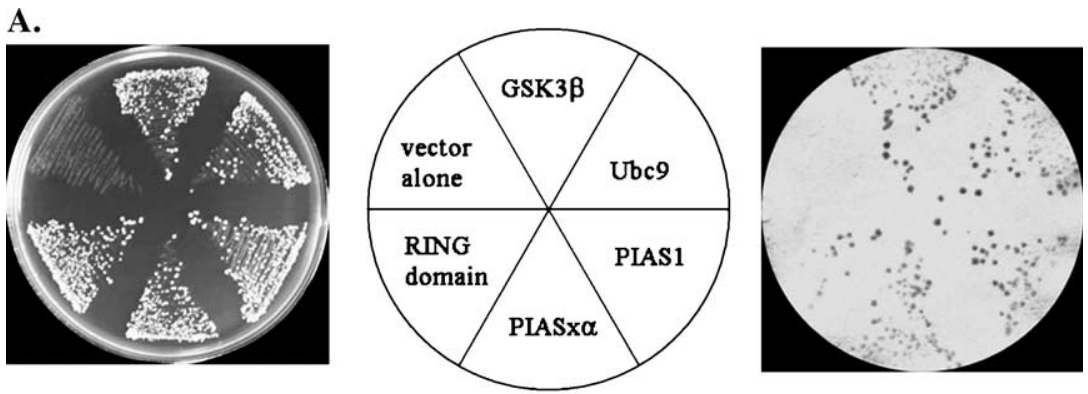

B.

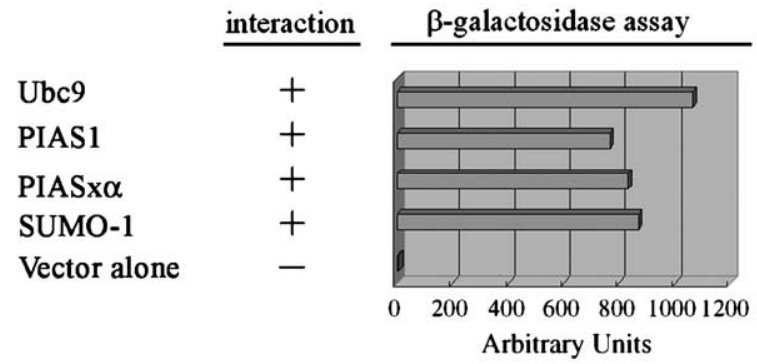

C.

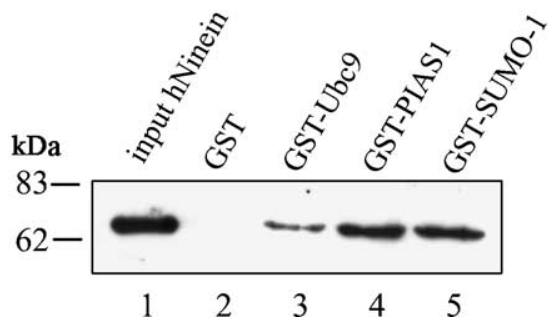

Fig. 1. Interactions between hNinein and SUMO-1. (A) Interactions between hNinein and GSK3 $\beta$, Ubc9 and PIAS in yeast two-hybrid screen. Yeast strain YRG2 was cotransformed with pAS2-1-C-hNinein and pACT2-GSK3 $\beta$, pACT2-Ubc9, pACT2-PIAS1, pACT2-PIASx $\alpha$, and pACT2-RING domain (PIASx $\alpha$ ) (middle panel). Transformed YRG2 cells containing both plasmids were streaked out on plates lacking tryptophan, leucine, and histidine in the presence of 5 mM 3 AT (left panel). Yeast cells were tested for $\beta$-galactosidase activity by a filter assay (right plane). (B) Interactions between hNinein and SUMO-1 in a yeast two-hybrid system. "+" indicates positive interaction; "-" indicates negative interaction. $\beta$-Galactosidase activity was determined by a liquid assay shown in the right panel. Units used are arbitrary units. (C) In vitro GST-pull down assays. His-tagged C-terminal hNinein prepared from E coli BL21(DE3) expressed and purified (lane 1) was added to GST (lane 2) or GST-Ubc9 (lane 3) or GST-PIAS1 (lane 4) or GST-SUMO-1 (lane 5) bound to glutathione-Sepharose beads. Proteins on the beads were finally analyzed by immunoblot analysis with anti-His antibody. The molecular marker shown on the left as indicated. 
A.

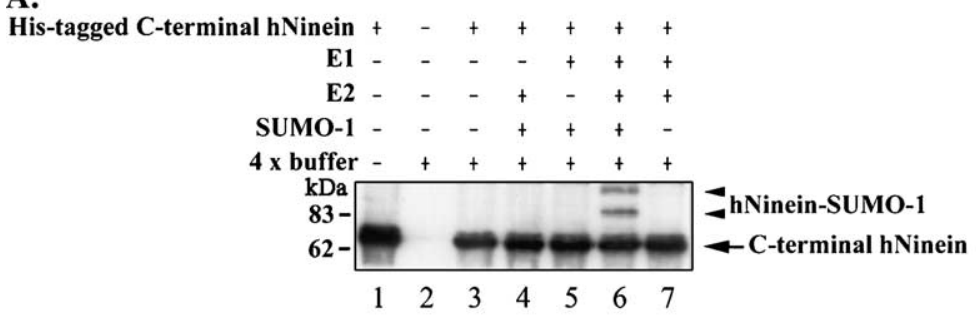

B.

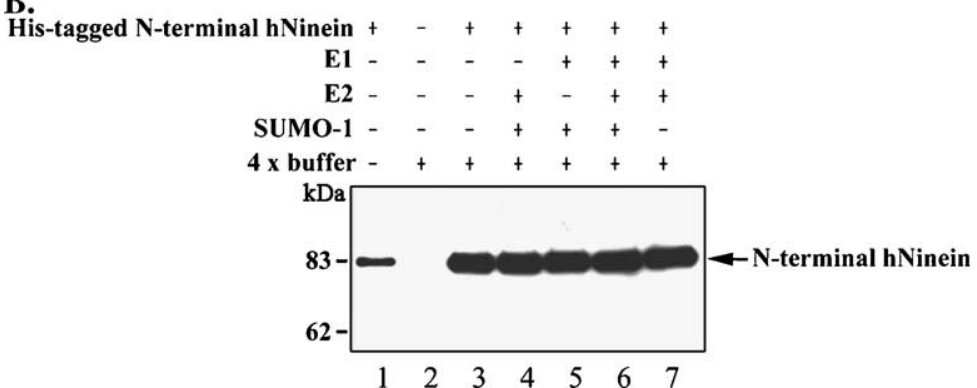

C.

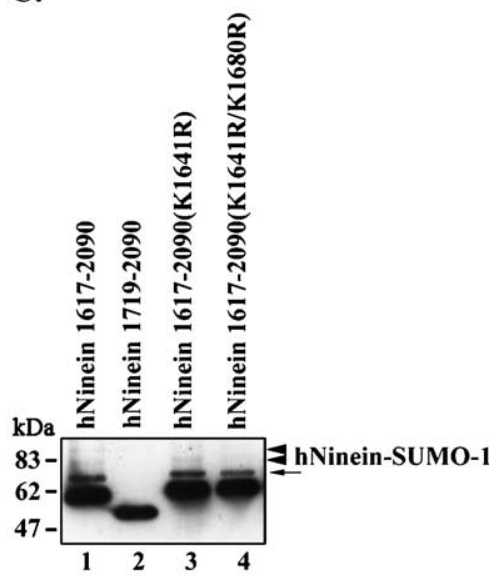

Fig. 2. SUMO-1 conjugation to hNinein in vitro and in vivo. Conjugation of hNinein by SUMO-1 was analyzed in vitro with either in the presence (+) or absence (-) of the assay mixtures containing purified His-tagged hNinein, E1, E2 (GST - Ubc9), and SUMO-1. The reactions were performed at $37^{\circ} \mathrm{C}$ for $2 \mathrm{~h}$ and SUMO1-hNinein in the reaction mixtures were finally analyzed by immunoblot analysis with anti-His antibody. (A) The His-tagged C-terminal hNinein, SUMO-1-hNinein conjugates are marked by arrowheads. Arrow indicates input His-tagged C-terminal hNinein. (B) The His-tagged N-terminal hNinein is not modified by SUMO-1. Arrow indicates input His-tagged N-terminal hNinein. (C) hNinein is covalently modified by SUMO-1 in vivo. HEK293 cells were transfected with pcDNA-C-terminal hNinein (aa. 1617-2090) (lanes 1), pcDNA-C-terminal hNinein (aa. 1719-2090) (lane 2), single mutant K1641R (lanes 3), or double mutant K1641R/K1680R (lanes 4). PolySUMOylated conjugates are marked by arrowheads. Arrow indicates non-specific band. The molecular marker is shown to the left of the blots.

bacterially expressed His-tagged C-terminal hNinein (aa. 1617-2090), N-terminal hNinein (aa. 1-471), and Histagged SUMO-1 lacking the four C-terminal amino acids (aa. 1-97) were purified as a substrate in the presence of recombinant SUMO-1, Ubc9 (as E2), and SUMO-1-activating E1. The full length of the SUMO-1 contains 101 amino acids, the protein lacking the four C-terminal amino acids still contains the glycine residues act as an active form in vitro assay system. SUMO-1 has a calculated size of $11 \mathrm{kDa}$ but SUMO-1 conjugated proteins run in the gel as if they would have additional about $15 \mathrm{kDa}$. As shown in Fig. 2A, adding E1, Ubc9 (E2), and SUMO-1 to the 73-kDa Histagged C-terminal hNinein translated in vitro resulted in the formation of two SUMO-1-hNinein conjugates $(88 \mathrm{kDa}$ and $103 \mathrm{kDa}$ ), detectable by immunoblot analysis (Fig. 2A, lane 6), indicating that hNinein is SUMO-1 modified into at least two forms of hNinein. In contrast, the N-terminal region of hNinein (aa. 1-471) was not conjugated by SUMO-1, since a band with a size larger than the target protein was unobserved (Fig. 2B). Meanwhile, at least two bands larger than the C-terminal hNinein fragment (aa. 1617-2090) were also detected in cell lysate by immunoblot analysis (Fig. 2C, lane1), indicating that hNinein is probably poly-SUMOylated. However, hNinein peptide fragment from amino acid 1719 to 2090 was not SUMOylated (Fig. 2C, lane 2). Changing the lysine residue at amino acid position 1641 to arginine [K1641R] and position both 1641/1680 to arginine [K1641R/K1680R] generated peptides that could either reduce or not be conjugated by SUMO-1 in vivo (Fig. $2 \mathrm{C}$, lanes 3 and 4), indicating that both K1641 and K1680 are SUMOylation sites. Taken together, these results provide compelling evidence that SUMO-1 was modified on Cterminal region of hNinein.

\section{SUMO promotes centrosomal proteins to nuclear localization}

To evaluate the subcellular localization of $\mathrm{hNinein}$ and SUMO-1, we expressed them ectopically in HeLa cells. hNinein and SUMO-1 were visualized by fluorescence by virtue of their GFP or RFP tags. Under our conditions, hNinein was expressed dominantly at the centrosome (Fig. 3A). Under

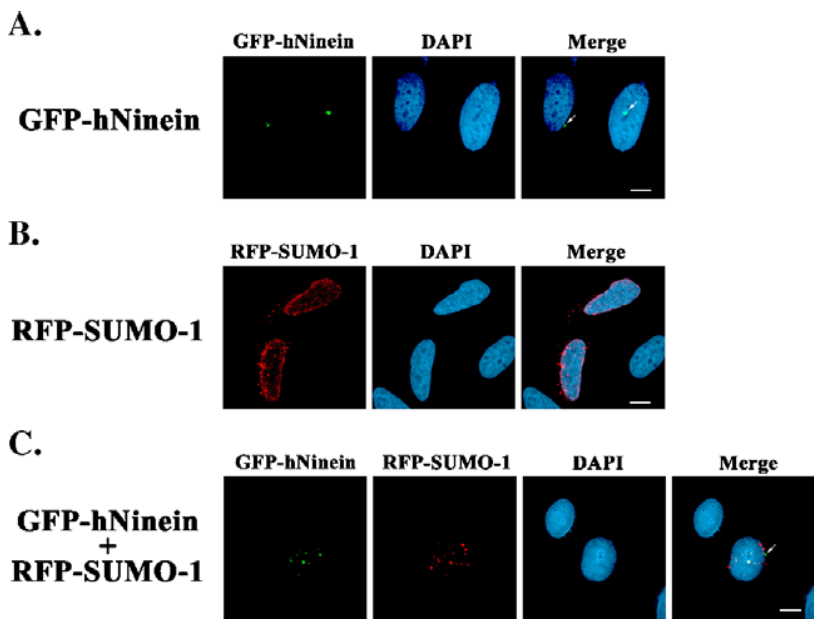

Fig. 3. hNinein partially associate with SUMO-1 in nucleus. HeLa cells were transfected with GFP-hNinein expression plasmid (A); RFP-SUMO-1 (B); or cotransfected with both $(C)$. Nuclei were stained with DAPI. The merging of GFP, RFP and DAPI images are shown at the far right. Arrows indicate hNinein localization at centrosome. Bars, $10 \mu \mathrm{m}$. 

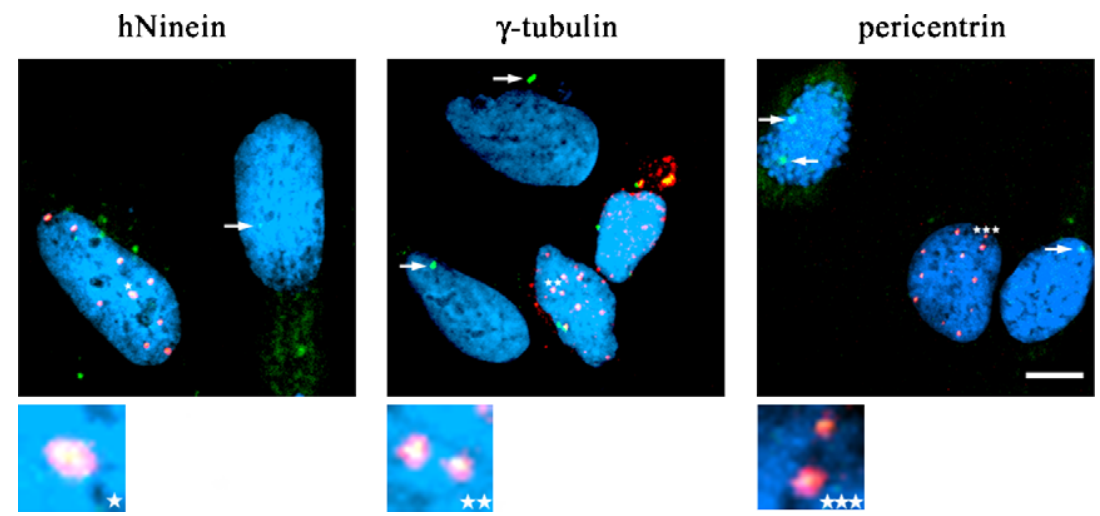

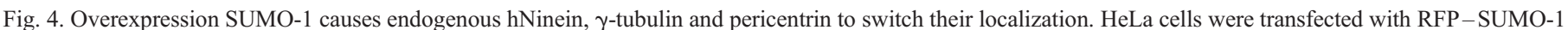

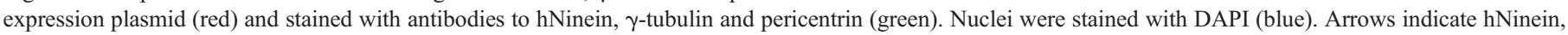

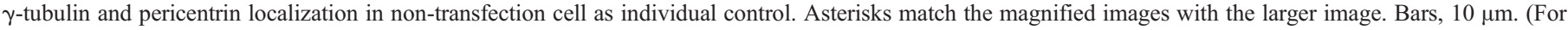
interpretation of the references to colour in this figure legend, the reader is referred to the web version of this article.)

the same conditions, SUMO-1 was expressed in the nucleus (Fig. 3B). Although partial associate was observed when both were co-expressed, a significant amount hNinein was translocated into nucleus (Fig. 3C). Moreover, overexpression SUMO-1 also causes endogenous hNinein, $\gamma$-tubulin and pericentrin were translocated into nucleus (Fig. 4). However, we investigated the subcellular distribution of null-mutant (K1641/1680R) of hNinein is similar to that observed for the wild type protein (data not shown), suggest that SUMO-1 functions to tether hNinein into nucleus may through SUMOylation or noncovalent protein-protein interaction. These results indicate that SUMO-1 could promote centrosomal proteins to nuclear localization.

\section{Discussion}

SUMO-1 is known to covalently modify a wide range of proteins via a three-step enzymatic pathway analogous to that of the ubiquitin pathway (Wilson and Rangasamy, 2001). Posttranscriptional modification by SUMO influences proteinprotein interactions, localization, and stability (Ahn et al., 2001; Joseph et al., 2002; Pichler et al., 2002; Marx, 2005). In this study, we observe that both covalent conjugation and a direct protein-protein interaction occur between the $\mathrm{C}$ terminal region of $\mathrm{hNinein}$ and SUMO-1, but do not occur in the $\mathrm{N}$-terminal region of $\mathrm{hNinein}$ (Fig 2A and B). Analysis of the amino acid sequence of the $\mathrm{C}$-terminal region of hNinein, revealed six putative consensus SUMOylation motifs ( $\psi \mathrm{KXX}$ ) at positions $1641,1680,1744,1800$ and 1909. Our data show that the two SUMO-1 moieties attached to hNinein C-terminus most likely represent a poly SUMO-1 chain at K1641 and K1680 two sites. Since this region also contains multiple functional domains, including centrosomal targeting, regulating and GSK3 $\beta$ binding domains (Hong et al., 2000a; Chen et al., 2003), SUMOylation may be crucial for the functions of hNinein. We speculate the dissociation of hNinein from mitotic centrosomes during M-phase may be regulated by localized phosphorylation rather than ubiquitin-proteasome proteolysis. Since the C-terminal region of hNinein is conjugated by SUMO-1, which may ultimately prevent the protein from ubiquitin-mediated degradation by $26 \mathrm{~S}$ proteasome (Fabunmi et al., 2000), suggesting that dissociation of hNinein from the mitotic centrosomes may be a result of protein phosphorylation instead of degradation, a phenomenon also observed for another centrosomal protein, C-Nap1 (Mayor et al., 2002). However, unlike the majority of ubiquitinated proteins, acceptors of SUMO-1 modifications are not targeted for degradation by 26S proteasome (Matunis et al., 1996; Mahajan et al., 1997; Ahn et al., 2001; Joseph et al., 2002). In this study, we show that hNinein interacts with Ubc9, PIAS family and SUMO-1, suggesting that SUMOylation may be involved in hNinein stability. In addition, we also show that hNinein interacts with PIAS family using PIAS RING fingerlike domain (Fig 1A). The PIAS family's ability to stimulate protein SUMOylation and the interaction with SUMOylated proteins are dependent on the conserved PIAS RING fingerlike domain (Kotaja et al., 2002). SUMO-1 modification of certain substrates is also known to have implications upon subcellular location (Joseph et al., 2002; Melchior et al., 2003). Reportedly, the centrosome proteins centrin and pericentrin, but not $\gamma$-tubulin, AKAP450, or hNinein, undertake a nucleocytoplasmic exchange as they concentrate in nucleus upon Ran G-protein (Keryer et al., 2003). Here we show that co-transfection hNinein and SUMO-1 cause a significant amount of GFP-hNinein translocated into nucleus (Fig. 3C). In addition, we also show that overexpression SUMO-1 causes a significant amount of endogenous hNinein, $\gamma$-tubulin and pericentrin to be translocated into nucleus (Fig. 4). These results indicate that SUMOylation may promote hNinein and probably also other centrosomal proteins to switch their localization, suggesting the importance of the SUMOylation may be a common phenomenon regulating in the centrosome activity. Further study will be needed to determine whether SUMOylation influences the function of hNinein and affects centrosomal location during the cell cycle. In conclusion, our data indicate that SUMO-1 may be involved in the centrosome activity through a SUMOylated centrosome structure protein, hNinein. Moreover, SUMO-1 may also promote other centrosomal proteins, such as $\gamma$-tubulin and pericentrin, which may also be involved in nucleocytoplasmic exchange, suggest- 
ing that the centrosome organization activity could be regulated by SUMOylation.

\section{Acknowledgments}

We thank Dr. S.T. Liu for helpful comments and suggestions. This work was supported by NSC 93-2314-B-037-015 (Taiwan, R.O.C.) to SLH and NSC 93-2320-B-037-037 to YRH.

\section{References}

Ahn, J.H., Xu, Y., Jang, W.J., Matunis, M.J., Hayward, G.S., 2001. Evaluation of interactions of human cytomegalovirus immediate-early IE2 regulatory protein with small ubiquitin-like modifiers and their conjugation enzyme Ubc9. Journal of Virology 75, 3859-3872.

Baron, A.T., Greenwood, T.M., Bazinet, C.W., Salisbury, J.L., 1992. Centrin is a component of the pericentriolar lattice. Biology of the Cell 76, 383-388.

Bouckson-Castaing, V., Moudjou, M., Ferguson, D.J., Mucklow, S., Belkaid, Y., Milon, G., Crocker, P.R., 1996. Molecular characterisation of ninein, a new coiled-coil protein of the centrosome. Journal of Cell Science 109, $179-190$

Bornens, M., 2002. Centrosome composition and microtubule anchoring mechanisms. Current Opinion in Cell Biology 14, 25-34.

Chang, L.K., Lee, Y.H., Cheng, T.S., Hong, Y.R., Lu, P.J., Wang, J.J., Wang, W.H., Kuo, C.W., Li, S.S., Liu, S.T., 2004. Post-translational modification of Rta of Epstein-Barr virus by SUMO-1. Journal of Biological Chemistry 279, 38803-38812.

Chen, C.H., Howng, S.L., Cheng, T.S., Chou, M.H., Huang, C.Y., Hong, Y.R., 2003. Molecular characterization of human ninein protein: two distinct subdomains required for centrosomal targeting and regulating signals in cell cycle. Biochemical and Biophysical Research Communications 308, 975-983.

Chien, C.T., Bartel, P.L., Sternglanz, R., Fields, S., 1991. The two-hybrid system: a method to identify and clone genes for proteins that interact with a protein of interest. Proceedings of the National Academy of Sciences of the United States of America 88, 9578-9582.

Dictenberg, J.B., Zimmerman, W., Sparks, C.A., Young, A., Vidair, C., Zheng, Y., Carrington, W., Fay, F.S., Doxsey, S.J., 1998. Pericentrin and gammatubulin form a protein complex and are organized into a novel lattice at the centrosome. Journal of Cell Biology 141, 163-174.

Doxsey, S.J., Stein, P., Evans, L., Calarco, P.D., Kirschner, M., 1994. Pericentrin, a highly conserved centrosome protein involved in microtubule organization. Cell 76, 639-650.

Doxsey, S., 2001. Re-evaluating centrosome function. Nature Reviews. Molecular Cell Biology 2, 688-698.

Fabunmi, R.P., Wigley, W.C., Thomas, P.J., DeMartino, G.N., 2000. Activity and regulation of the centrosome-associated proteasome. Journal of Biological Chemistry 275, 409-413.

Fields, S., Song, O., 1989. A novel genetic system to detect protein-protein interactions. Nature 340, 245-246.

Fuller, S.D., Gowen, B., Reinsch, S., Sawyer, A., Buendia, B., Wepf, R., Karsenti, E., 1995. The core of the mammalian centriole contains gammatubulin. Current Biology 51, 384-393.

Gong, L., Li, B., Millas, S., Yeh, E.T., 1999. Molecular cloning and characterization of human AOS1 and UBA2, components of the centrinactivating enzyme complex. FEBS Letters 448, 185-189.

Hong, Y.R., Chen, C.H., Chang, J.H., Wang, S.K., Sy, W.D., Chou, C.K., Howng, S.L., 2000. Cloning and characterization of a novel human ninein protein that interacts with the glycogen synthase kinase 3beta. Biochimica et Biophysica Acta 1492, 513-516.

Hong, Y.R., Chen, C.H., Chuo, M.H., Liou, S.Y., Howng, S.L., 2000. Genomic organization and molecular characterization of the human ninein gene. Biochemical and Biophysical Research Communications 279, 989-995.

Johnson, E.S., Gupta, A.A., 2001. An E3-like factor that promotes SUMO conjugation to the yeast septins. Cell 106, 735-744.
Joseph, J., Tan, S.H., Karpova, T.S., McNally, J.G., Dasso, M., 2002. SUMO-1 targets RanGAP1 to kinetochores and mitotic spindles. Journal of Cell Biology 156, 595-602.

Kahyo, T., Nishida, T., Yasuda, H., 2001. Involvement of PIAS1 in the sumoylation of tumor suppressor p53. Molecular Cell 8, 713-718.

Keryer, G., Di Fiore, B., Celati, C., Lechtreck, K.F., Mogensen, M., Delouvee, A., Lavia, P., Bornens, M., Tassin, A.M., 2003. Part of Ran is associated with AKAP450 at the centrosome: involvement in microtubule-organizing activity. Molecular Biology of the Cell 14, 4260-4271.

Kotaja, N., Karvonen, U., Janne, O.A., Palvimo, J.J., 2002. PIAS proteins modulate transcription factors by functioning as SUMO-1 ligases. Molecular Biology of the Cell 22, 5222-5234.

Mack, G.J., Rees, J., Sandblom, O., Balczon, R., Fritzler, M.J., Rattner, J.B., 1998. Autoantibodies to a group of centrosomal proteins in human autoimmune sera reactive with the centrosome. Arthritis and Rheumatism $41,551-558$.

Mahajan, R., Delphin, C., Guan, T., Gerace, L., Melchior, F., 1997. A small ubiquitin-related polypeptide involved in targeting RanGAP1 to nuclear pore complex protein RanBP2. Cell 88, 97-107.

Mahajan, R., Gerace, L., Melchior, F., 1998. Molecular characterization of the SUMO-1 modification of RanGAP1 and its role in nuclear envelope association. Journal of Cell Biology 140, 259-270.

Matunis, M.J., Coutavas, E., Blobel, G., 1996. A novel ubiquitin-like modification modulates the partitioning of the Ran-GTPase-activating protein RanGAP1 between the cytosol and the nuclear pore complex. Journal of Cell Biology 135, 1457-1470.

Marx, J., 2005. Cell biology. SUMO wrestles its way to prominence in the cell. Science 307, 836-839.

Mayor, T., Hacker, U., Stierhof, Y.D., Nigg, E.A., 2002. The mechanism regulating the dissociation of the centrosomal protein C-Nap1 from mitotic spindle poles. Journal of Cell Science 115, 3275-3284.

Melchior, F., Schergaut, M., Pichler, A., 2003. SUMO: ligases, isopeptidases and nuclear pores. Trends in Biochemical Sciences 28, 612-618.

Middendorp, S., Paoletti, A., Schiebel, E., Bornens, M., 1997. Identification of a new mammalian centrin gene, more closely related to Saccharomyces cerevisiae CDC31 gene. Proceedings of the National Academy of Sciences of the United States of America 94, 9141-9146.

Mogensen, M.M., 1999. Microtubule release and capture in epithelial cells. Biology of the Cell 91, 331-341.

Moudjou, M., Paintrand, M., Vigues, B., Bornens, M., 1991. A human centrosomal protein is immunologically related to basal body-associated proteins from lower eucaryotes and is involved in the nucleation of microtubules. Journal of Cell Biology 115, 129-140.

Pichler, A., Gast, A., Seeler, J.S., Dejean, A., Melchior, F., 2002. The nucleoporin RanBP2 has SUMO1 E3 ligase activity. Cell 108, 109-120.

Piel, M., Meyer, P., Khodjakov, A., Rieder, C.L., Bornens, M., 2000. The respective contributions of the mother and daughter centrioles to centrosome activity and behavior in vertebrate cells. Journal of Cell Biology 149, $317-330$.

Rangasamy, D., Woytek, K., Khan, S.A., Wilson, V.G., 2000. SUMO-1 modification of bovine papillomavirus E1 protein is required for intranuclear accumulation. Journal of Biological Chemistry 275, 37999-38004.

Schwarz, S.E., Matuschewski, K., Liakopoulos, D., Scheffner, M., Jentsch, S., 1998. The ubiquitin-like proteins SMT3 and SUMO-1 are conjugated by the UBC9 E2 enzyme. Proceedings of the National Academy of Sciences of the United States of America 95, 560-564.

$\mathrm{Su}, \mathrm{H} ., \mathrm{Li}, \mathrm{S}$., 2002. Molecular features of human ubiquitin-like SUMO genes and their encoded proteins. Gene 296, 65.

Wilson, V.G., Rangasamy, D., 2001. Intracellular targeting of proteins by sumoylation. Experimental Cell Research 271, 57-65.

Zheng, Y., Jung, M.K., Oakley, B.R., 1991. Gamma-tubulin is present in Drosophila melanogaster and Homo sapiens and is associated with the centrosome. Cell 65, 817-823.

Zhu, L., 1997. Yeast GAL4 two-hybrid system. A genetic system to identify proteins that interact with a target protein. Methods in Molecular Biology 63, 173-196. 